Western University

Scholarship@Western

Brain and Mind Institute Researchers'

Publications

Brain and Mind Institute

$4-1-2021$

\title{
Skin and muscle receptors shape coordinated fast feedback responses in the upper limb
}

Christopher J. Forgaard

The University of Western Ontario

Sasha Reschechtko

The University of Western Ontario

Paul L. Gribble

The University of Western Ontario, pgribble@uwo.ca

J. Andrew Pruszynski

The University of Western Ontario

Follow this and additional works at: https://ir.lib.uwo.ca/brainpub

Part of the Neurosciences Commons, and the Psychology Commons

\section{Citation of this paper:}

Christopher J Forgaard, Sasha Reschechtko, Paul L Gribble, J Andrew Pruszynski, Skin and muscle receptors shape coordinated fast feedback responses in the upper limb, Current Opinion in Physiology, Volume 20, 2021, Pages 198-205, ISSN 2468-8673, https://doi.org/10.1016/j.cophys.2021.02.001.

(https://www.sciencedirect.com/science/article/pii/S2468867321000328) 


\section{Skin and muscle receptors shape coordinated fast feedback responses in the upper limb Christopher J Forgaard ${ }^{1,2}$, Sasha Reschechtko $1,3,4$, Paul L Gribble ${ }^{1,2,3,5}$ and J Andrew Pruszynski ${ }^{1,2,3,4,5}$}

\begin{abstract}
Despite many real-world examples where skin and muscle receptors must function in concert to support movement control, responses based on these sensory modalities are usually separated from one another in laboratory studies. Proprioception is often considered the domain of muscle receptors, whereas the skin's function is often assumed to be discriminative touch. This distinction understates the extent to which sensory feedback from skin and muscle work together to shape successful movement control. Here we review the functional characteristics and similarities between fast feedback responses of the upper limb originating from muscle stretch and skin slip. We place an emphasis on recent evidence of their highly inter-dependent nature and how they build on one another to implement common tasks like object manipulation in the face of external forces applied to the arm or hand.
\end{abstract}

\footnotetext{
Addresses

${ }^{1}$ Brain and Mind Institute, Western University, London, Ontario, Canada

${ }^{2}$ Dept. of Psychology, Western University, Canada

${ }^{3}$ Dept. of Physiology and Pharmacology, Western University, Canada

${ }^{4}$ Robarts Research Institute, Western University, Canada
}

Corresponding author: Forgaard, Christopher J (cforgaar@uwo.ca)

${ }^{5}$ Co-senior authorship.

Current Opinion in Physiology 2021, 21:xx-yy

This review comes from a themed issue on Proprioception

Edited by Tim Cope and Leah Bent

https://doi.org/10.1016/j.cophys.2021.02.001

2468-8673/@ 2021 Elsevier Ltd. All rights reserved.

\section{Introduction}

Holding onto an object in a dynamic environment requires constant adjustments to grip force and arm orientation. Following a sudden bump to the arm or pull on the object, the CNS rapidly activates finger muscles to increase grip force and muscles throughout the arm to stabilize or adjust the limb to a desired orientation. These 'fast feedback responses'-which occur earlier than humans can voluntarily respond to an external stimulus (i.e. $<100 \mathrm{~ms}$ ) - are elicited by cutaneous receptors detecting object slip and muscle receptors activated by stretch. Despite many real-world scenarios where sensory feedback from both skin and muscle receptors must be rapidly integrated to successfully maintain grasp, laboratory investigations of grip responses (triggered by object slip) and stretch reflexes (evoked by muscle stretch) are typically conducted separately. This historical separation partly stems from stretch reflex studies typically investigating proximal muscles whereas slip studies typically focus on the hand. In this review, we consider how sensory feedback from mechanoreceptors in the glabrous skin of the fingertips and muscle receptors throughout the upper limb function together to produce coordinated fast feedback responses to maintain an object in grasp.

\section{Fast feedback responses to mechanical stimuli}

Fast feedback responses evoked by stretching upper limb muscles are classically divided into two epochs in the stretched muscle (Figure 1). Occurring first is the shortlatency response (SLR: $\sim 20-50 \mathrm{~ms}$ ) which results from activation of Ia afferents acting over a spinal pathway $[1,2]$. Immediately following is the long-latency response (LLR; 50-100 ms) produced by continued engagement of spinal pathways via Ia and other afferents and input from supraspinal pathways $\left[3,4,5^{\circ}\right]$. The supraspinal component includes a transcortical pathway involving primary motor cortex (M1), shown in humans via noninvasive neural recordings [6] and brain stimulation [7], and electrophysiology and lesion studies in non-human primates [7-9]. Other supraspinal regions contribute including dorsal premotor cortex [9], supplementary motor area [10], posterior parietal cortex [9], cerebellum [11], and reticular formation [12,13].

The SLR is functionally limited and relatively inflexible, though it can be modified according to changes in body configuration [14] or with extensive training [15,16]. Presumably because the LLR is generated in part by supraspinal circuits, it can be strongly influenced by many factors including the volitional intent of the participant $[17,18]$. For example, individuals can modulate the LLR according to verbal instruction of how to respond to the perturbation $[3,19,20]$, as well as visuospatial goals [20-22]. The classic manipulation involves asking participants to either 'resist' or 'let go' upon receiving the perturbation. The resist instruction results in a large LLR whereas the response is reduced and sometimes even fully inhibited on let go trials [3]. Sophistication of the LLR is further shown by its ability to scale 


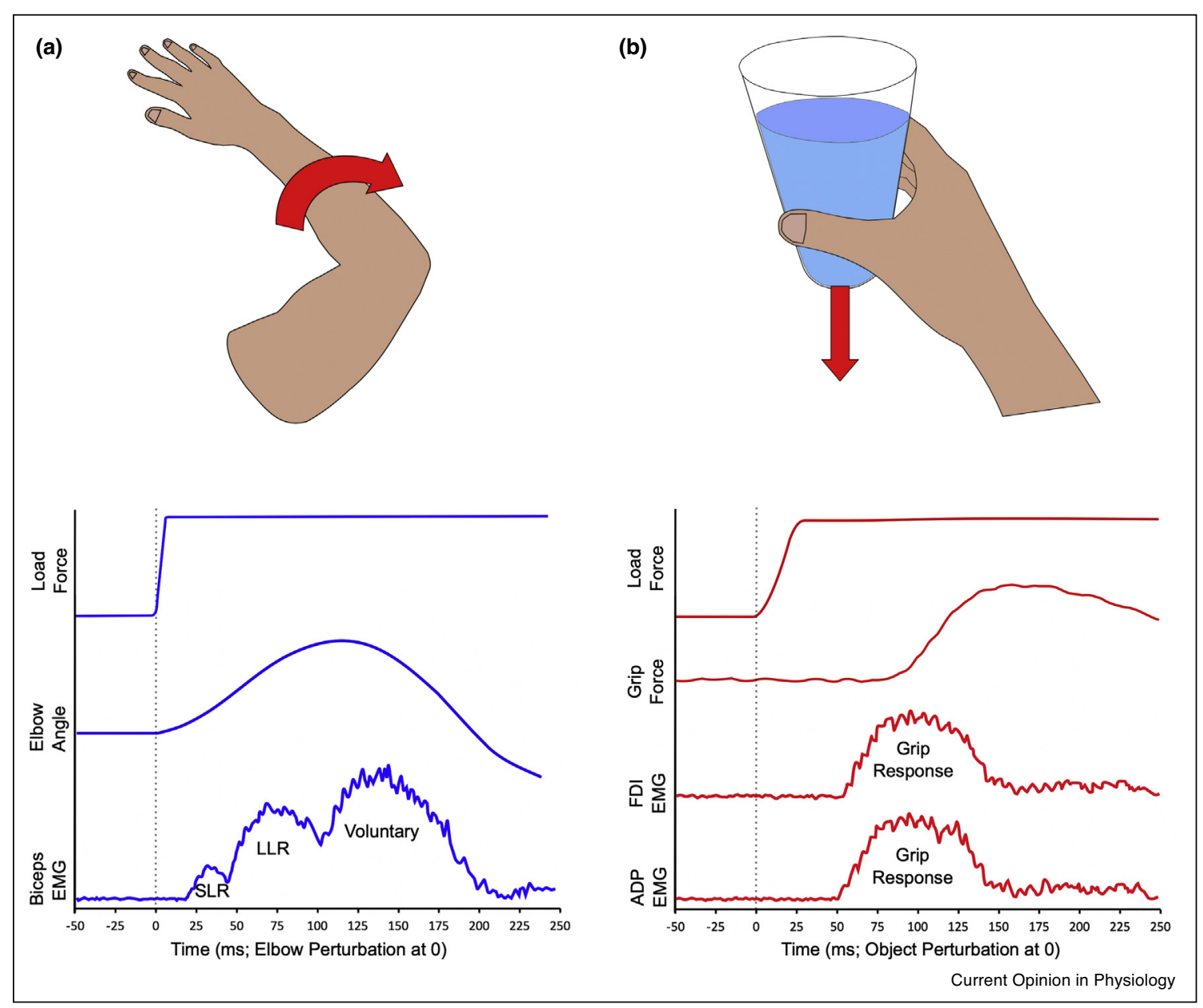

Muscle responses evoked by mechanical perturbations.

(a) Cartoon of a typical mechanical perturbation causing rapid elbow extension. A short-latency (SLR) response appears in the biceps brachii EMG recording between $20-50 \mathrm{~ms}$, followed by a long-latency response (LLR; $50-100 \mathrm{~ms}$ ), and voluntary activity (>100 ms). The evoked muscle activity counteracts the induced motion from the perturbation, ultimately producing elbow flexion. (b) Cartoon of a typical downward mechanical perturbation to a cup held in grasp. The rapid increase in object load force against the fingertips induces slip of the cup. A grip response (50$150 \mathrm{~ms}$ ) appears in intrinsic and extrinsic hand muscles contributing to grip force production. Shown here is EMG activity from first dorsal interossei (FDI) and adductor pollicis (ADP), hand muscles commonly recorded in grip response investigations [32,51 $\left.{ }^{\circ}\right]$.

continuously with intended movement distance [22], intersegmental limb dynamics [23-26] including arm orientation [21], and ongoing decisional processes [27-30].

When holding an object in precision grip, rapid changes in the force that the object exerts against the fingertips elicits a fast feedback response in intrinsic and extrinsic hand muscles that increase grip force (e.g. first dorsal interossei, flexor pollicis brevis, adductor pollicis, flexor digitorum superficialis) [31,32]. This grip response (see Figure 1b) originates from cutaneous low-threshold mechanoreceptors detecting slip against the fingertips
[31,33-36]. Given the similar latency to the LLR, it makes sense to wonder whether a transcortical route involving M1 also underlies this response. Studies using EEG [37], fMRI [38], transcranial magnetic stimulation [34], and single-cell recordings [39] all support the involvement of M1. However, while M1 can influence the grip response once underway [34], modulation of corticospinal excitability occurs after the grip response has been initiated $[33,34]$ and the response is largely preserved in patients with impaired contralateral corticospinal projections from M1 [40]. The evidence thus suggests that the grip response is likely initiated subcortically [33] possibly involving cerebellum $[38,41,42]$ and/ 
or spinal circuits [ $\left.43-46,47^{\circ}\right]$. More evidence for a subcortical origin comes from the finding that the grip response seems less sensitive to arbitrary modulation than the LLR: rather, it optimally functions to prevent dropping objects across a range of situations. For example, a stronger response is evoked when holding a more slippery object [48], following faster slip perturbations [31,32], or slips in more precarious directions (i.e. with gravity or away from the hand [49]). However, unlike the LLR, the grip response is not sensitive to verbal instruction: asking participants to "let go" of an object upon perceiving the slip stimulus has minimal influence over the rapid activation of finger/thumb flexors and concomitant increase in grip force [50].

Despite a traditional distinction that skin receptors underlie the grip response and muscle spindles produce the LLR - most limb or handheld object perturbations, especially during object manipulation, activate both receptor types. Cutaneous receptors display positional sensitivity albeit with lower fidelity than muscle receptors $\left[52,53,54^{\circ}\right]$ and single cutaneous (FAI, FAII, and SAII) afferents (but not spindle afferents) have been shown to have strong coupling with motoneurons innervating the hand $[55,56]$. In investigations of stretch reflexes, cutaneous receptors are also activated as forces are applied to the hand or arm to induce joint rotation [2,57,cf.58]. Moreover, the grip response can also be evoked by mechanically stretching finger [59] or thumb flexor muscles [60]. Depending on the behavioural goal or postural constraints, sensory feedback from upper limb muscle and skin afferents can also be flexibly routed to produce fast feedback responses in many muscles throughout the perturbed $\left[21,23,32,61,62^{\bullet \bullet}\right]$, contralateral [42,63-67], and even lower limbs [65,68].

\section{Coordinating feedback across the arm and hand}

Mechanical perturbations during object manipulation can manifest as either external forces acting on the object or directly on the arm. Relative motion between the object and hand can thus arise from at least two distinct movement patterns. In one scenario, the object is pulled away from your hand-as when your dog takes off running while you hold the leash. This causes slip against the fingers while forces from the leash are also transmitted throughout the arm. Alternatively, a perturbation delivered directly to your arm, such as when holding a cup of coffee, results not only in arm muscle stretch, but also finger slip against the object due to the object's inertia. In order to compensate for these perturbations, we need to make corrections to hand position (by moving the arm) and to grip force (to hold onto the object).

One approach to understanding these complex interactions is to experimentally dissociate object slip from forces transmitted to the arm. Hernandez-Castillo et al. $\left[62^{\circ}\right]$ recently investigated how tactile feedback from the fingertips is routed to produce responses in proximal arm muscles. These authors showed that a finger slip stimulus can affect the fast feedback response in shoulder flexor and extensor muscles-but that this relationship is specific to the relative directions of object slip and arm movement. When the stimulus mimicked an object slipping out of the hand, participants responded more quickly by moving their arm in the same direction as the slip (i.e. by activating the clavicular head of pectoralis major), as if they were moving to keep their hand on the object. In addition, they showed that the LLR observed in shoulder muscles is modified by the presence of a slip stimulus at the fingertips. On these trials, a shoulder extension perturbation was always delivered-but critically was sometimes also paired with a finger slip stimulus in the opposite direction (i.e. out of the hand). Participants showed a larger response in the pectoralis muscle to the combined shoulder perturbation and finger slip stimuli (compared to a perturbation-only stimulus), the scenario mimicking what would happen if that torque were pulling their arm off of a handheld object. Together, these results indicate that afferent feedback from the fingers can be quickly integrated with proprioceptive feedback from the arm to direct rapid, behaviourally relevant responses. An important topic for future investigation is understanding how different regions such as the thalamus or primary sensory cortex integrate somatosensory and proprioceptive feedback in order to contribute to fast feedback response modulation $\left[62^{\bullet \bullet}, 69\right]$.

As discussed previously, the LLR displays a level of task dependency similar to voluntary control. Another recent study probed whether goal-dependent modulation occurs between the LLR in arm muscles and grip response when moving an object to different spatial goals [51 $\left.{ }^{\circ \bullet}\right]$. In this study, mechanical perturbations were delivered to the shoulder and displaced the arm either towards a target (requiring minimal intervention; Figure 2, IN target, blue profiles) or away from a target (requiring vigorous intervention; Figure 2, OUT target, red profiles). Perturbation direction was not known in advance. Following perturbations that displaced the shoulder into flexion, goal-dependent LLR modulation was observed in the posterior deltoid both with and without the presence of a handheld object (Figure 2d). On trials where participants held an object using a precision grip, the shoulder perturbation also evoked a grip response in hand muscles (first dorsal interossei, flexor pollicis brevis, and adductor pollicis; See Figure 2c). Importantly, this grip response exhibited goal-dependent modulation simultaneously with modulation of the LLR in posterior deltoid (beginning at $60 \mathrm{~ms}$ ): the more vigorous arm movements following a perturbation away from a target produced larger inertial load forces against the fingertips, and participants showed a stronger grip response on these trials to prevent the object from slipping away. 


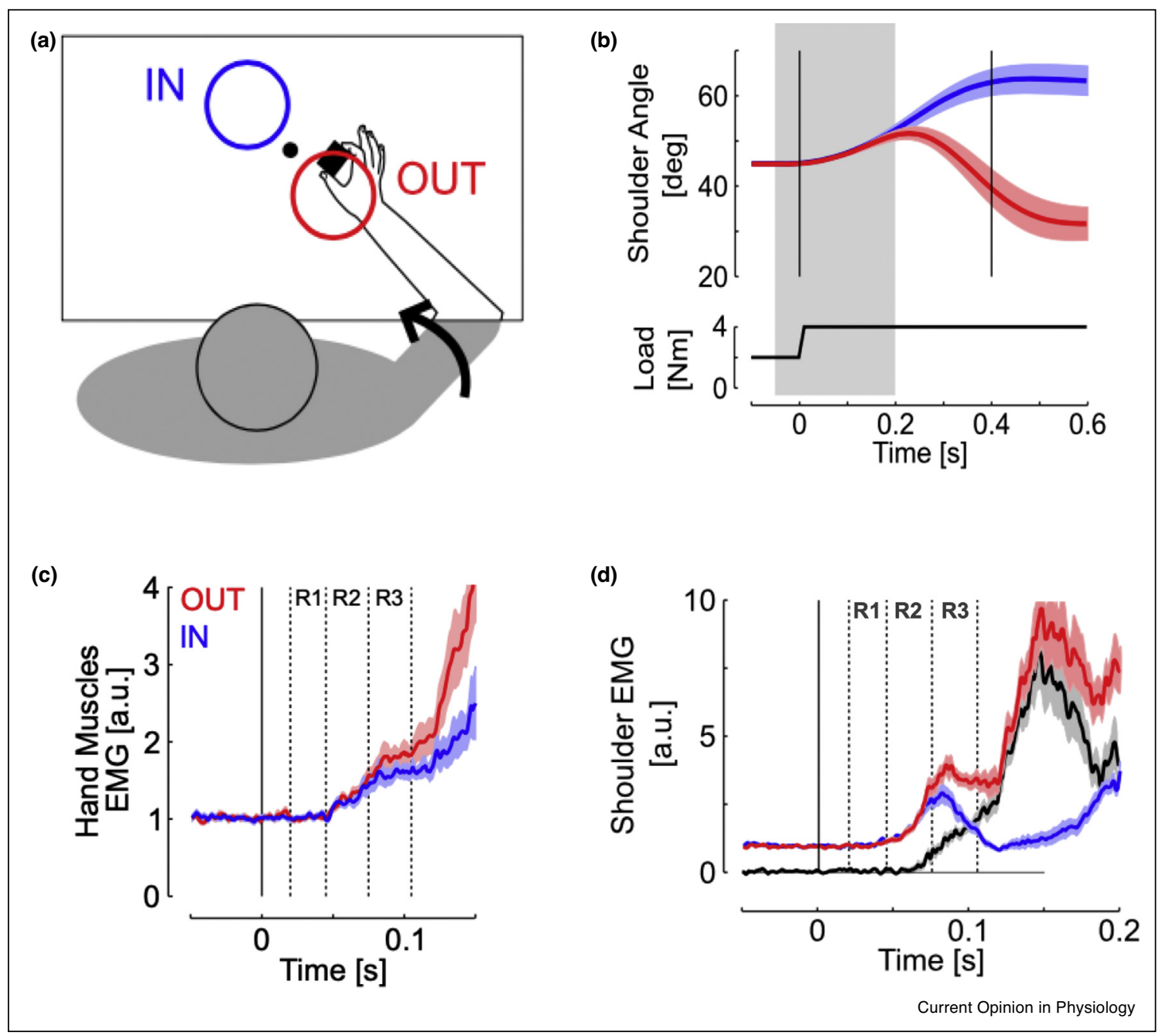

Coordination of fast feedback responses between the arm and hand.

(a) Experimental setup where a shoulder flexion torque is applied and participants either allow this perturbation to move their hand into a target (IN, blue) or they counteract the torque by extending the shoulder and moving in the opposite direction to a target (OUT, red). Note that an object is held between the index finger and thumb. Also note that perturbation direction was unknown in advance. This figure only shows data from shoulder flexion perturbation trials. (b) Elbow displacement data for the two target conditions. (c) Hand muscle activity (averaged signal from first dorsal interossei, adductor pollicis, and flexor pollicis brevis). R1 refers to the SLR epoch. R2 is the first half of the LLR epoch (45-75 ms). R3 is the second half of the LLR epoch $(75-105 \mathrm{~ms})$. (d) Shoulder muscle (posterior deltoid) EMG activity. Black profile represents the difference between OUT and IN. Reproduced with permission from Ref. [51 ${ }^{\circ}$ ].

At first glance, these results from Crevecoeur et al. [51 $\left.{ }^{\bullet \bullet}\right]$ appear to fit well with observations of coordination between arm movements and grasp during self-initiated object manipulation [70,71]. It is commonly proposed that the CNS predicts the outcome of upcoming actions including the object load forces that arise as a result of voluntary arm movements [72]. This internal prediction allows the CNS to modulate grip forces in parallel with changing load forces against the fingertips. However, if this explanation were to hold for the reactive control of arm and hand action, it would predict either a delay in the modulation of grip response with respect to the posterior deltoid LLR, or a delay in both responses. This is because generation of the modulated grip response motor commands must await internal predictions generated from the production of modulated shoulder extensor motor commands. Additionally, the CNS must account for the fact that efferent signals have to travel a further distance to reach intrinsic hand muscles, a delay estimated at $\sim 10 \mathrm{~ms}$ relative to posterior deltoid $\left[51^{\circ \bullet}\right]$. It is important to emphasize that neither of these outcomes were observed by Crevecoeur $e t a l$. [51 ${ }^{\circ}$ ]. While hand and arm responses 
occurred simultaneously, they were not delayed with respect to arm movements performed when the fingers were not holding onto an object.

One possible explanation for this observation is that the CNS fully pre-plans hand responses for multiple possible arm actions and then selects an appropriate response after the perturbation has begun. Although this may be a tenable strategy in experimental conditions where participants know the perturbation they may experience, it is less likely when considering the great variety of possible arm and hand perturbations we experience in everyday life, and the apparent behavioural and directional specificity of observed arm and grasp responses. It is also inconsistent with the rapid flexibility of upper limb fast feedback responses following unpredictable arm perturbations [22,73]. As an alternative to fully pre-planning multiple hand responses, it was suggested that during the movement planning phase (before a perturbation), the CNS couples together motor commands for hand and arm muscles $\left[51^{\bullet \bullet}\right]$. Critically, part of the planning process also involves specifying how sensory feedback will be rapidly transformed into motor output to efficiently achieve the behavioural goal. While supraspinal regions have been the primary target for investigations into the source(s) of fast feedback response flexibility, coordination between the LLR and the grip response may also rely on descending modulatory commands onto spinal circuits that rapidly integrate proprioceptive and cutaneous inputs.

An important target for future research is identification of the neural circuitry permitting rapid coordination of fast feedback responses between the arm and hand, such as in the object manipulation studies described in this review. Numerous studies have highlighted a transcortical pathway through M1 as a source of goal-dependent LLR modulation in upper-limb muscles [7,9,74]. Because of the strong monosynaptic corticospinal projections onto hand motoneurons, it is also commonly suggested that M1 has an important role in controlling dexterous finger movements [75]. Interestingly, evidence suggests that spinal premotor interneurons are also critical for grasp control $\left[43,44,46,47^{\circ}, 76\right]$ and it was recently shown that these spinal neurons are strongly activated by a mechanical finger stretch perturbation at an appropriate latency to contribute to the LLR in finger muscles $\left[5^{\circ}\right]$. However, to our knowledge, the role of spinal interneurons on the grip response following object slip has not been reported. Given that premotor interneurons in cervical spinal cord receive input from somatosensory and proprioceptive afferents $\left[5^{\circ}, 47^{\circ}\right]$, descending signals from higher centres [77], and make divergent connections onto multiple intrinsic hand muscles [44], they are a prime candidate to contribute to goal-dependent modulation of the grip response. Exactly how premotor interneurons may support goal-dependent grip modulation simultaneously with LLR modulation in proximal arm muscles remains unresolved and should be investigated in future experiments.

In addition to identifying the neural circuitry coordinating the grip response and the LLR, it is important to investigate feedback response coordination in scenarios that more closely mimic normal behaviour. Most studies conducted on the grip response involve objects held in precision grip [33]; however, in real-world scenarios, we often interact with objects using power grip or multi-finger prismatic grasp. Recent work has shown different neural control between grip types [78-81], and appropriate responses to a slipping object might differ when the thumb is not used in opposition to the other digits. Coordination of responses might also differ because power grip appears well-conserved over an evolutionary timescale, whereas precision grip is thought to rely on phylogenetically newer brain structures and descending pathways that exist only in some dexterous primates (including humans) [82].

While this review has focused on fast feedback responses in the upper limb, future work should also consider drawing comparisons to responses in the lower limb. Fast feedback responses evoked by mechanical postural perturbations also rely on the integration of cutaneous feedback and proprioceptive feedback from muscle receptors [83], and many postural reactions can be evoked by cutaneous stimulation of the leg or foot [84]. Highlighting similarities and differences between limbs may lead to a better understanding of the specialized role different sources of feedback have in subserving various fast feedback responses.

The LLR and the grip response are two of the fastest expressions of goal-directed behaviour. Despite their close relationship in many ecological activities, they have been studied separately for many years, implicitly reinforcing the view that muscle receptors are solely responsible for the LLR whereas cutaneous receptors are only engaged in the grip response. We are now beginning to understand that these responses are in fact highly inter-dependent and it is important to consider both the role skin receptors play in shaping the LLR and the effect muscle spindles have on the grip response.

\section{Conflict of interest statement}

Nothing declared.

\section{Acknowledgements}

This work was supported by grants from the Natural Sciences and Engineering Research Council of Canada (NSERC), Canadian Institutes of Health Research (CIHR), Western BrainsCAN, and Canada First Research Excellence Fund (CFREF). JAP received a salary award from the Canada Research Chairs program. 


\section{References and recommended reading}

Papers of particular interest, published within the period of review, have been highlighted as:

- of special interest

$\bullet$ of outstanding interest

1. Pierrot-Deseilligny E, Burke D: The Circuitry of the Human Spinal Cord: Its Role in Motor Control and Movement Disorders. Cambridge University Press; 2005.

2. Reschechtko S, Pruszynski JA: Stretch reflexes. Curr Biol 2020, 30:R1025-R1030.

3. Hammond PH: The influence of prior instruction to the subject on an apparently involuntary neuro-muscular response. $J$ Physiol 1956, 132:17-18.

4. Lourenço G, Iglesias C, Cavallari P, Pierrot-Deseilligny E, Marchand-Pauvert $V$ : Mediation of late excitation from human hand muscles via parallel group II spinal and group I transcortical pathways. J Physiol 2006, 572:585-603.

5. Soteropoulos DS, Baker SN: Long-latency responses to a - mechanical perturbation of the index finger have a spinal component. J Neurosci 2020, 40:3933-3948

This study demonstrated that cervical spinal interneurons respond to mechanical stretch of index finger. Critically, many premotor interneurons fired at an appropriate time to contribute to the LLR.

6. MacKinnon CD, Verrier MC, Tatton WG: Motor cortical potentials precede long-latency EMG activity evoked by imposed displacements of the human wrist. Exp Brain Res 2000, 131:477-490.

7. Pruszynski JA, Kurtzer I, Nashed JY, Omrani M, Brouwer B, Scott SH: Primary motor cortex underlies multi-joint integration for fast feedback control. Nature 2011, 478:387-390.

8. Cheney PD, Fetz EE: Corticomotoneuronal cells contribute to long-latency stretch reflexes in the rhesus monkey. J Physiol 1984, 349:249-272.

9. Omrani M, Murnaghan CD, Pruszynski JA, Scott SH: Distributed task-specific processing of somatosensory feedback for voluntary motor control. eLife 2016, 5 http://dx.doi.org/10.7554/ eLife.13141.

10. Spieser L, Aubert S, Bonnard M: Involvement of SMAp in the intention-related long latency stretch reflex modulation: a TMS study. Neuroscience 2013, 246:329-341.

11. Kurtzer I, Trautman P, Rasquinha RJ, Bhanpuri NH, Scott SH, Bastian AJ: Cerebellar damage diminishes long-latency responses to multijoint perturbations. J Neurophysiol 2013, 109:2228-2241.

12. Heckman RL, Perreault EJ: Uncertainty in when a perturbation will arrive influences the preparation and release of triggered responses. Exp Brain Res 2019, 237:2353-2365.

13. Zonnino A, Farrens AJ, Ress D, Sergi F: Measurement of stretchevoked brainstem function using fMRI. bioRxiv $2020 \mathrm{http}: / / \mathrm{dx}$ doi.org/10.1101/2020.06.19.161315 https://www.biorxiv.org/ content/10.1101/2020.06.19.161315v1 abstract.

14. Weiler J, Gribble PL, Pruszynski JA: Spinal stretch reflexes support efficient hand control. Nat Neurosci 2019, 22:529-533.

15. Norton JJS, Wolpaw JR: Acquisition, maintenance, and therapeutic use of a simple motor skill. Curr Opin Behav Sci 2018, 20:138-144

16. Wolpaw JR, Braitman DJ, Seegal RF: Adaptive plasticity in primate spinal stretch reflex: initial development. $J$ Neurophysiol 1983, 50:1296-1311.

17. Pruszynski JA, Scott SH: Optimal feedback control and the long-latency stretch response. Exp Brain Res 2012, 218:341359.

18. Scott SH: A functional taxonomy of bottom-up sensory feedback processing for motor actions. Trends Neurosci 2016, 39:512-526.
19. Forgaard CJ, Franks IM, Maslovat D, Chua R: Influence of kinesthetic motor imagery and effector specificity on the longlatency stretch response. J Neurophysiol 2019, 122:2187-2200.

20. Forgaard CJ, Franks IM, Maslovat D, Chin L, Chua R: Voluntary reaction time and long-latency reflex modulation. $J$ Neurophysiol 2015, 114:3386-3399.

21. Weiler J, Gribble PL, Pruszynski JA: Rapid feedback responses are flexibly coordinated across arm muscles to support goaldirected reaching. J Neurophysiol 2018, 119:537-547.

22. Pruszynski JA, Kurtzer I, Scott SH: Rapid motor responses are appropriately tuned to the metrics of a visuospatial task. J Neurophysiol 2008, 100:224-238.

23. Maeda RS, Gribble PL, Pruszynski JA: Learning new feedforward motor commands based on feedback responses. Curr Biol 2020, 30:1941-1948.e3.

24. Maeda RS, Cluff T, Gribble PL, Pruszynski JA: Feedforward and feedback control share an internal model of the arm's dynamics. J Neurosci 2018, 38:10505-10514.

25. Kurtzer IL, Pruszynski JA, Scott SH: Long-latency reflexes of the human arm reflect an internal model of limb dynamics. Curr Biol 2008, 18:449-453.

26. Weiler J, Saravanamuttu J, Gribble PL, Pruszynski JA: Coordinating long-latency stretch responses across the shoulder, elbow, and wrist during goal-directed reaching. $J$ Neurophysiol 2016, 116:2236-2249.

27. Selen LPJ, Shadlen MN, Wolpert DM: Deliberation in the motor system: reflex gains track evolving evidence leading to a decision. J Neurosci 2012, 32:2276-2286.

28. Nashed JY, Crevecoeur F, Scott SH: Influence of the behavioral goal and environmental obstacles on rapid feedback responses. J Neurophysiol 2012, 108:999-1009.

29. Nashed JY, Crevecoeur F, Scott SH: Rapid online selection between multiple motor plans. J Neurosci 2014, 34:1769-1780.

30. Yang L, Michaels JA, Pruszynski JA, Scott SH: Rapid motor responses quickly integrate visuospatial task constraints. Exp Brain Res 2011, 211:231-242.

31. Cole KJ, Abbs JH: Grip force adjustments evoked by load force perturbations of a grasped object. J Neurophysiol 1988, 60:1513-1522.

32. Johansson RS, Westling G: Programmed and triggered actions to rapid load changes during precision grip. Exp Brain Res 1988, 71:72-86.

33. Johansson RS: Somatosensory signals and sensorimotor transformations in reactive control of grasp. Somesthesis and the Neurobiology of the Somatosensory Cortex. 1996:271-282.

34. Johansson RS, Lemon RN, Westling G: Time-varying enhancement of human cortical excitability mediated by cutaneous inputs during precision grip. J Physiol 1994, 481:761-775.

35. Johansson RS, Hger C, Bäckström L: Somatosensory control of precision grip during unpredictable pulling loads. III. Impairments during digital anesthesia. Exp Brain Res 1992, 89:204-213.

36. Macefield V Hager-Ross C, Johansson R: Control of grip force during restraint of an object held between finger and thumb: responses of cutaneous afferents from the digits. Exp Brain Res 1996, 108:155-171.

37. Kourtis D, Kwok HF, Roach N, Wing AM, Praamstra P: Maintaining grip: anticipatory and reactive EEG responses to load perturbations. J Neurophysiol 2008, 99:545-553.

38. Ehrsson $\mathrm{HH}$, Fagergren A, Ehrsson GO, Forssberg $\mathrm{H}$ : Holding an object: neural activity associated with fingertip force adjustments to external perturbations. J Neurophysiol 2007, 97:1342-1352

39. Picard N, Smith AM: Primary motor cortical responses to perturbations of prehension in the monkey. J Neurophysiol 1992, 68:1882-1894. 
40. Harrison LM, Mayston MJ, Johansson RS: Reactive control of precision grip does not depend on fast transcortical reflex pathways in X-linked Kallmann subjects. J Physiol 2000, 527:641-652.

41. Dugas C, Smith AM: Responses of cerebellar Purkinje cells to slip of a hand-held object. J Neurophysiol 1992, 67:483-495.

42. White O, Dowling N, Bracewell RM, Diedrichsen J: Hand interactions in rapid grip force adjustments are independent of object dynamics. J Neurophysiol 2008, 100:2738-2745.

43. Takei T, Seki K: Spinal premotor interneurons mediate dynamic and static motor commands for precision grip in monkeys. Neurosci 2013, 33:8850-8860.

44. Takei T, Seki K: Spinal interneurons facilitate coactivation of hand muscles during a precision grip task in monkeys. $J$ Neurosci 2010, 30:17041-17050.

45. Tazoe T, Rothwell JC, Perez MA: Subcortical control of precision grip after human spinal cord injury. J Neurosci 2014 34:7341-7350 https://www.jneurosci.org/content/34/21/7341. short.

46. Bui TV, Akay T, Loubani O, Hnasko TS, Jessell TM, Brownstone RM: Circuits for grasping: spinal dl3 interneurons mediate cutaneous control of motor behavior. Neuron 2013 78:191-204.

47. Oya T, Takei T, Seki K: Distinct sensorimotor feedback loops for - dynamic and static control of primate precision grip. Commun Biol 2020, 3:156

Oya et al. demonstrated that spinal premotor interneurons display beta band coherence with upper-limb EMG during dynamic object manipulation. Beta band coherence between M1 and EMG was restricted to static grip.

48. Cole KJ, Johansson RS: Friction at the digit-object interface scales the sensorimotor transformation for grip responses to pulling loads. Exp Brain Res 1993, 95:523-532.

49. Häger-Ross C, Cole KJ, Johansson RS: Grip-force responses to unanticipated object loading: load direction reveals body- and gravity-referenced intrinsic task variables. Exp Brain Res 1996, 110:142-150.

50. Winstein CJ, Abbs JH, Petashnick D: Influences of object weight and instruction on grip force adjustments. Exp Brain Res 1991 87:465-469.

51. Crevecoeur F, Thonnard J-L, Lefèvre P, Scott SH: Long-latency

-. feedback coordinates upper-limb and hand muscles during object manipulation tasks. eNeuro 2016, 3

These authors found that a shoulder mechanical perturbation also elicited a grip response in hand muscles when holding an object was in precision grip. Remarkably, the grip response displayed goal-dependent modulation simultaneous with the shoulder LLR.

52. Edin BB: Quantitative analysis of static strain sensitivity in human mechanoreceptors from hairy skin. J Neurophysiol 1992, 67:1105-1113.

53. Edin B: Cutaneous afferents provide information about knee joint movements in humans. J Physiol 2001, 531:289-297.

54. Smith L, Norcliffe-Kaufmann L, Palma J-A, Kaufmann H,

- Macefield VG: Elbow proprioception is normal in patients with a congenital absence of functional muscle spindles. J Physiol 2020, 598:3521-3529

This study showed that patients with an absence of functional muscle spindles have partially preserved proprioception around the elbow joint. It would be interesting to test whether skin afferents in these patients can also be used to generate an LLR following a mechanical arm perturbation.

55. McNulty PA, Macefield VG: Modulation of ongoing EMG by different classes of low-threshold mechanoreceptors in the human hand. J Physiol 2001, 537:1021-1032.

56. McNulty PA, Türker KS, Macefield VG: Evidence for strong synaptic coupling between single tactile afferents and motoneurones supplying the human hand. J Physiol 1999 518:883-893.
57. Bawa P, McKenzie DC: Contribution of joint and cutaneous afferents to longer-latency reflexes in man. Brain Res 1981, 211:185-189.

58. Wolpaw JR: Correlations between task-related activity and responses to perturbation in primate sensorimotor cortex. $J$ Neurophysiol 1980, 44:1122-1138.

59. Crevecoeur F, Barrea A, Libouton X, Thonnard J-L, Lefèvre P: Multisensory components of rapid motor responses to fingertip loading. J Neurophysiol 2017, 118:331-343.

60. Cole KJ, Gracco VL, Abbs JH: Autogenic and nonautogenic sensorimotor actions in the control of multiarticulate hand movements. Exp Brain Res 1984, 56:582-585.

61. Gielen CC, Ramaekers L, Van Zuylen EJ: Long-latency stretch reflexes as co-ordinated functional responses in man. $J$ Physiol 1988, 407:275-292.

62. Hernandez-Castillo CR, Maeda RS, Pruszynski JA, Diedrichsen J:

- Sensory information from a slipping object elicits a rapid and automatic shoulder response. J Neurophysiol 2020, 123:11031112

This study showed a slip stimulus at the fingertips produced a fastfeedback response in shoulder muscles that moved the arm in the direction of slip. When paired with a mechanical shoulder perturbation this somatosensory feedback facilitated the shoulder LLR.

63. Khong KYW, Galán F, Soteropoulos DS: Rapid crossed responses in an intrinsic hand muscle during perturbed bimanual movements. J Neurophysiol 2020, 123:630-644.

64. Omrani M, Diedrichsen J, Scott SH: Rapid feedback corrections during a bimanual postural task. J Neurophysiol 2013, 109:147-161

65. Marsden CD, Merton PA, Morton HB: Human postura responses. Brain 1981, 104:513-534.

66. Reschechtko S, Pruszynski JA: Voluntary modification of rapid tactile-motor responses during reaching differs from its visuomotor counterpart. J Neurophysiol 2020, 124:284-294.

67. Pruszynski JA, Johansson RS, Flanagan JR: A rapid tactilemotor reflex automatically guides reaching toward handheld objects. Curr Biol 2016, 26:788-792.

68. Lowrey CR, Nashed JY, Scott SH: Rapid and flexible whole body postural responses are evoked from perturbations to the upper limb during goal-directed reaching. J Neurophysiol 2017, 117:1070-1083.

69. Kim SS, Gomez-Ramirez M, Thakur PH, Hsiao SS: Multimodal interactions between proprioceptive and cutaneous signals in primary somatosensory cortex. Neuron 2015, 86:555-566.

70. Wolpert DM, Flanagan JR: Motor prediction. Curr Biol 2001, 11 R729-732.

71. Johansson RS, Flanagan JR: Coding and use of tactile signals from the fingertips in object manipulation tasks. Nat Rev Neurosci 2009, 10:345-359.

72. Flanagan JR, Wing AM: The role of internal models in motion planning and control: evidence from grip force adjustments during movements of hand-held loads. J Neurosci 1997 17:1519-1528.

73. Pruszynski JA, Kurtzer I, Scott SH: The long-latency reflex is composed of at least two functionally independent processes. $J$ Neurophysiol 2011, 106:449-459.

74. Pruszynski JA, Omrani M, Scott SH: Goal-dependent modulation of fast feedback responses in primary motor cortex. J Neurosci 2014, 34:4608-4617.

75. Lemon RN: Descending pathways in motor control. Annu Rev Neurosci 2008, 31:195-218.

76. Takei T, Seki K: Synaptic and functional linkages between spinal premotor interneurons and hand-muscle activity during precision grip. Front Comput Neurosci 2013, 7:40.

77. Prut $Y$, Fetz EE: Primate spinal interneurons show premovement instructed delay activity. Nature 1999, 401:590-594 
78. Federico P, Perez MA: Distinct corticocortical contributions to human precision and power grip. Cereb Cortex 2017, 27:5070-5082.

79. Tazoe T, Perez MA: Cortical and reticular contributions to human precision and power grip. J Physiol 2017, 595:2715-2730.

80. Baker SN, Perez MA: Reticulospinal contributions to gross hand function after human spinal cord injury. J Neurosci 2017 , 37:9778-9784.

81. Honeycutt CF, Kharouta M, Perreault EJ: Evidence for reticulospinal contributions to coordinated finger movements in humans. J Neurophysiol 2013, 110:1476-1483.
82. Rathelot J-A, Strick PL: Subdivisions of primary motor cortex based on cortico-motoneuronal cells. Proc Natl Acad Sci U S A 2009, 106:918-923.

83. Ting LH: Dimensional reduction in sensorimotor systems: a framework for understanding muscle coordination of posture. Prog Brain Res 2007, 165:299-321.

84. Zaback M, Horslen BC, Cleworth TW, Collings L, Langlet C, Inglis JT, Carpenter MG: Influence of emotional stimuli on lower limb cutaneous reflexes during human gait. Neurosci Lett 2018 , 664:123-127. 